\title{
UC2 Light sheet microscope: From an education tool to a biological application
}

Barbora Marsikova $^{1,2}$, Benedict Diederich ${ }^{1,3}$, René Lachmann ${ }^{1,2}$, Rainer Heintzmann ${ }^{1,2,3}$

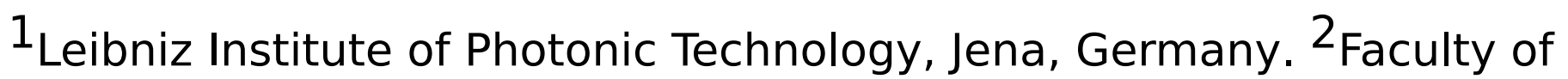
Physics and Astronomy, Friedrich Schiller University, Jena, Germany.

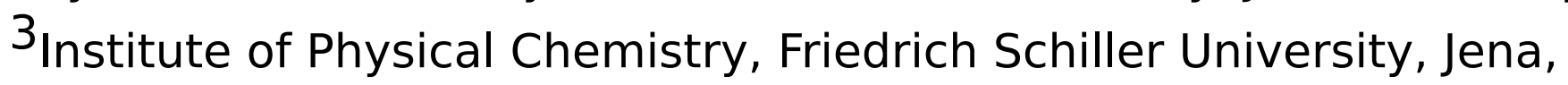
Germany

\section{Abstract Text}

The open-source optical toolbox UC2 [YouSeeToo] [1] simplifies the process of building optical setups, by combining 3D-printed cubes, each holding a specific component (e.g. lens, mirror) on a magnetic square-grid baseplate. The use of widely available consumables and 3D printing, together with documentation and software, offers an extremely low-cost and accessible alternative for both education and research areas. In order to reduce the entry barrier, we provide a fully comprehensive toolbox, TheBOX, that allows the user to go from brightfield to light sheet microscope within a few minutes, by simply exchanging some components. This is highly beneficial in the field, where one wants to try several imaging modalities on the same sample and in educational environments where optical concepts can be explained easily.

In the previous LSFM conference we presented our simplest selective plane illumination microscope, that is easily portable and reproducible, thanks to the use of of-the-shelf-components and with the price tag around 400 EUR. It proved itself to be a valuable tool for demonstrating the principles of this method to users, who might work with a light sheet system all the time but never looked inside the black box.

In the next step, we move from the classroom to the lab and focus on the scientific application of our light sheet setup. We decided to 
change the configuration to the rather unusual open-top SPIM [2], where the optics is located underneath the sample. This makes the microscope ideal for classical samples mounted with a coverslip or, for example, for microfluidic chips. In the microfluidic chip, the cells grow in conditions that simulate the in-vivo situation. For observing these samples, it is crucial to not disturb the conditions and therefore a compact light sheet microscope that doesn't have any special requirements for sample mounting seems to be the ideal solution.

The project is in a work-in-progress state and aims to grow along with an active community of interested researchers, biologists, teachers but also other enthusiastic users and makes microscopy affordable, portable, understandable and generally accessible. 